\title{
A physically interpretable statistical wake steering model
}

\author{
Balthazar Sengers ${ }^{1}$, Matthias Zech ${ }^{2}$, Pim Jacobs ${ }^{1}$, Gerald Steinfeld ${ }^{1}$, and Martin Kühn ${ }^{1}$ \\ ${ }^{1}$ ForWind, Institute of Physics, Carl von Ossietzky University Oldenburg, Küpkersweg 70, 26129 Oldenburg, Germany \\ ${ }^{2}$ German Aerospace Center (DLR), Institute of Networked Energy Systems, Carl-von-Ossietzky-Str. 15, 26129 Oldenburg, \\ Germany
}

Correspondence: Balthazar Sengers (balthazar.sengers@uni-oldenburg.de)

\begin{abstract}
Wake steering models for control purposes are typically based on analytical wake descriptions tuned to match experimental or numerical data. This study explores the potential of a data-driven statistical wake steering model with a high degree of physical interpretation. A linear model trained with large eddy simulation data estimates wake parameters such as deficit, center location and curliness from measurable inflow and turbine variables. These wake parameters are then used

5 to generate vertical cross sections of the wake at desired downstream locations. In a validation against eight boundary layers ranging from neutral to stable conditions, the trajectory, shape and available power of the far wake are accurately estimated. The approach allows the choice of different input parameters, while the accuracy of the power estimates remains largely unchanged. A significant improvement in accuracy is shown in a benchmark study against two analytical wake models, especially under derated operating conditions and stable atmospheric stratifications. While results are encouraging, the model's sensitivity to training data needs further investigation.
\end{abstract}

\section{Introduction}

Wind turbine wakes cause considerable power losses and increased loads at downstream machines. Control strategies to mitigate these negative effects are gaining support in the wind energy community. In particular wake steering, or wake redirection through intentional yaw misalignment (Dahlberg and Medici, 2003; Wagenaar et al., 2012), is regarded as a promising control strategy. A yaw misalignment introduces a lateral thrust force component, redirecting the downstream wake and generating two counter-rotating vortices around lower and upper tip height that curl the wake into a kidney shape (Howland et al., 2016). Numerical simulations (e.g. Gebraad et al., 2016; Fleming et al., 2018; Hulsman et al., 2020), wind tunnel experiments (e.g. Campagnolo et al., 2016; Bartl et al., 2018; Bastankhah and Porté-Agel, 2019) and free field campaigns (e.g. Fleming et al., 2017, 2019, 2020; Bromm et al., 2018) have demonstrated the potential of an increased wind farm power production when utilizing the wake steering concept. The efficacy of wake steering is strongly dependent on turbine operation and atmospheric inflow characteristics, such as the turbine thrust coefficient (Jimenez et al., 2010), atmospheric stability (Vollmer et al., 2016), wind shear (Schottler et al., 2017) and turbulence intensity (Bastankhah and Porté-Agel, 2016). 
https://doi.org/10.5194/wes-2021-43

Preprint. Discussion started: 21 May 2021

(c) Author(s) 2021. CC BY 4.0 License.

\section{(c) (i)}

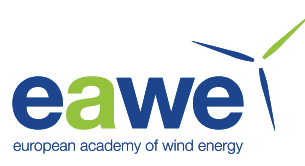

Wake steering controllers regulating the turbine yaw angle are often based on simple wake models that can describe the downstream wake under different inflow conditions. These models, such as those present in the FLORIS framework (NREL, 2020), are typically based on a simplified analytical description of the momentum conservation equations for stationary inflow conditions. When combined with a dynamic controller, wind direction variability can be included (Rott et al., 2018; Simley et al., 2020). The performance of these wake steering controllers, and therefore the accuracy of the underlying wake models, is essential for the application of wake steering as a control strategy in a real wind farm.

30 Frequently used in recent years is the wake model based on Gaussian self-similarity (Bastankhah and Porté-Agel, 2014, 2016; Abkar and Porté-Agel, 2015; Niayifar and Porté-Agel, 2016). Combining wake deficit and wake deflection models, the Gaussian (GAUS) model uses turbulence intensity as an atmospheric inflow parameter. It was validated against field measurements in Annoni et al. (2018) and used as a controller in a field campaign in Fleming et al. (2019). A disadvantage of this model is the negligence of the counter-rotating vortices generated with yaw misalignment and consequently the absence of wake curling. In addition, it does not account for the initial wake deflection caused by the torque induced wake rotation in sheared inflow (Zahle and Sørensen, 2008). The curl model (Martínez-Tossas et al., 2019) accounts for these phenomena by explicitly including vortices in a model based on linearized Reynolds-averaged Navier-Stokes equations. Having a strong physical basis, it is able to include a wide range of atmospheric conditions and allows flexibility in the wake shape generation. A disadvantage is the high computational expense compared to GAUS. Therefore, King et al. (2020) proposed to include the vortex description of the curl model into GAUS in the Gaussian-Curl Hybrid (GCH) model. This incorporates the initial wake deflection and even secondary wake steering, the deflection of the wake of a downstream turbine by the vortices generated by the yawed upstream turbine (Fleming et al., 2018), but not the curling of the wake itself. In addition, the model includes a wake recovery term representing added entrainment by the vortices generated due to yaw misalignment. Fleming et al. (2020) showed promising results when using GCH as controller input in a free field campaign.

These analytical models contain parameters that can be tuned to match numerical or experimental data. In addition, data can be used to formulate parameterized error terms (Schreiber et al., 2020). However, completely data-driven wake models remain rare and those that exist generally use complex machine learning models with a low interpretability (e.g. Göçmen and Giebel, 2018; Ti et al., 2020). This is remarkable, since simple statistical models are widely used for prediction purposes, including wind power (Stathopoulos et al., 2013; Messner and Pinson, 2019) and power curve predictions (Marčiukaitis et al., 2017).

50 The objective of this study is to explore the potential of a statistical wake steering model (SWSM) that is data-driven, but retains a high degree of physical interpretation. It is investigated whether the curled wake can accurately be described by a set of measurable inflow and turbine variables, and how the use of these variables can be optimized in an interpretable model. Next, the potential of such a model is systematically assessed by evaluating its performance with large eddy simulations (LES) for a reference wind speed under different atmospheric conditions and subsequently benchmarking it against two analytical wake models (GAUS and GCH). Lastly, the sensitivity of a statistical model to training data and minimal considerations before application as a wake model are discussed. 
Table 1. Summary of simulation parameters and classification into neutral (NBL), near neutral (NNBL), weakly stable (WSBL) and stable (SBL) boundary layers. The size $\left(L_{x, \mathrm{p}}, L_{y}, L_{z}\right)$ of the domains is normalized by the rotor diameter $(\mathrm{D}=126 \mathrm{~m})$. All parameters are identical in precursor and main simulations, except for the domain size which is extended in streamwise direction $\left(L_{x, \mathrm{~m}}\right) . t_{\mathrm{p}}$ is the simulated time of the precursor run, $u_{g}$ and $v_{g}$ the geostrophic wind, $\partial \theta \partial t^{-1}$ the heating rate, $H$ the sensible heat flux and $z_{0}$ the surface roughness length.

\begin{tabular}{llllllllllll}
\hline & & $t_{\mathrm{p}}$ & $L_{x, \mathrm{p}}$ & $L_{x, \mathrm{~m}}$ & $L_{y}$ & $L_{z}$ & $u_{g}$ & $v_{g}$ & $\partial \theta \partial t^{-1}$ & $H$ & $z_{0}$ \\
& & {$[\mathrm{~h}]$} & {$[\mathrm{D}]$} & {$[\mathrm{D}]$} & {$[\mathrm{D}]$} & {$[\mathrm{D}]$} & {$\left[\mathrm{m} \mathrm{s}^{-1}\right]$} & {$\left[\mathrm{m} \mathrm{s}^{-1}\right]$} & {$\left[\mathrm{K} \mathrm{h}^{-1}\right]$} & {$\left[\mathrm{K} \mathrm{m} \mathrm{s}^{-1}\right]$} & {$[\mathrm{m}]$} \\
\hline BL1 & NBL & 28 & 40.6 & 61.0 & 20.3 & 14.0 & 10.115 & -3.969 & - & - & 0.1 \\
BL2 & NBL & 28 & 40.6 & 61.0 & 20.3 & 14.0 & 10.595 & -5.572 & - & - & 0.5 \\
BL3 & WSBL & 25 & 27.9 & 50.0 & 14.0 & 8.4 & 9.952 & -5.115 & -0.125 & - & 0.1 \\
BL4 & WSBL & 45 & 27.9 & 50.0 & 14.0 & 8.4 & 10.607 & -6.447 & -0.125 & - & 0.5 \\
BL5 & SBL & 20 & 11.4 & 30.5 & 7.6 & 4.6 & 9.500 & -5.170 & -0.250 & - & 0.1 \\
BL6 & SBL & 20 & 11.4 & 30.5 & 7.6 & 4.6 & 10.565 & -6.585 & -0.250 & - & 0.5 \\
BL7 & NBL & 40 & 40.6 & 61.0 & 20.3 & 14.0 & 9.609 & -3.193 & - & - & 0.03 \\
BL8 & NNBL & 40 & 40.6 & 61.0 & 20.3 & 14.0 & 9.831 & -3.488 & - & -0.001 & 0.1 \\
\hline
\end{tabular}

\section{Large eddy simulations}

In this study data are generated with revision 3475 of the PArallelized Large eddy simulation Model (PALM, Maronga et al. (2020)), which uses a non-hydrostatic incompressible Boussinesq approximation of the Navier-Stokes equations and the Monin-Obukhov Similarity Theory to describe surface fluxes. A precursor without and a subsequent main simulation with one turbine make up the simulation chain.

\subsection{Precursor simulations}

Realistic turbulent inflow conditions are generated from an initially laminar flow by adding random perturbations in a precursor simulation with cyclic horizontal boundary conditions. A regularly spaced grid on a right-handed Cartesian coordinate system with $\Delta=5 \mathrm{~m}$ is used in the boundary layer, while at higher altitudes the vertical grid size increases with $6 \%$ per cell to save computational costs. The Coriolis parameter corresponds to $55^{\circ} \mathrm{N}$ and default numerical schemes are used. To study the potential of a statistical wake steering model under different inflow conditions, eight boundary layers (BLs) ranging from a neutral to a strongly stable BL are used as reference inflow conditions, all having approximately the same wind speed and direction at hub height. As reported by Vollmer et al. (2016), wake steering is ineffective in a convective boundary layer and is therefore not considered in this study. The total simulation time and domain size are determined empirically until convergence to a stationary state occurs and are dependent on the size of the largest eddies that explicitly need to be resolved. The details of the precursor simulations are summarized in Table 1.

BL1 and BL2 portray neutral conditions with roughness lengths representing low crops $\left(z_{0}=0.1 \mathrm{~m}\right)$ and parkland $\left(z_{0}=0.5 \mathrm{~m}\right)$, which are typical landscapes found in Northern Germany. Following Basu et al. (2008), constant cooling rates at the surface are 

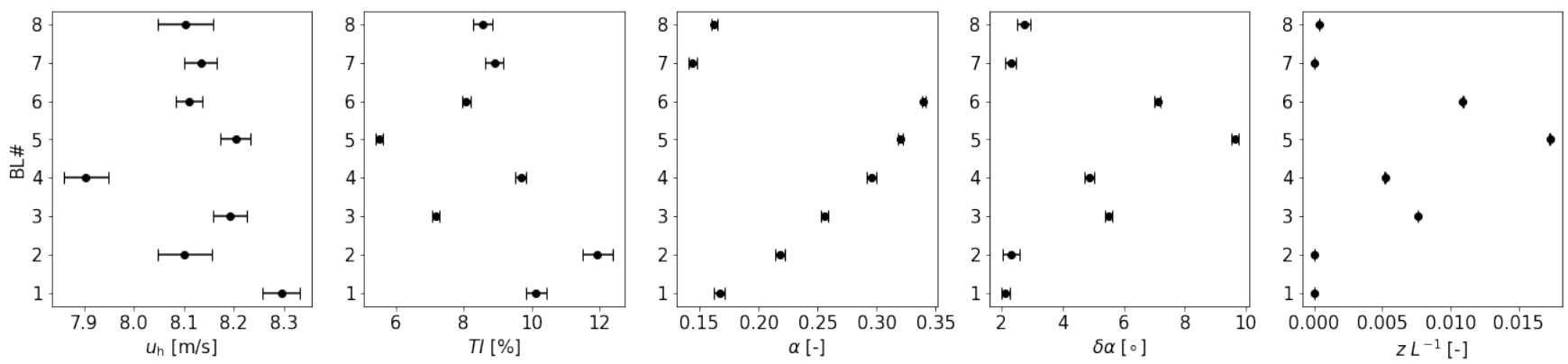

Figure 1. Summary of the most relevant inflow parameters (60 min averages), given as mean (dots) and standard deviation (whiskers) over the 15 main simulations. Considered are wind speed $\left(u_{\mathrm{h}}\right)$ and turbulence intensity at hub height $(T I)$, wind shear $(\alpha)$ and veer $(\delta \alpha)$ over the rotor area and the Obukhov stability parameter $\left(z L^{-1}\right)$ at the $2.5 \mathrm{~m}$. Equations for these variables can be found in Table 3 .

75 prescribed to generate stable BLs, where BL3 and BL4 represent weakly stable ( $\partial \theta \partial t^{-1}=-0.125 \mathrm{~K} \mathrm{~h}^{-1}$ ), and BL5 and BL6 strongly stable conditions ( $\partial \theta \partial t^{-1}=-0.25 \mathrm{~K} \mathrm{~h}^{-1}$, following Beare and Macvean (2004)). Two additional (near) neutral BLs are generated, one representing grassland $\left(z_{0}=0.03 \mathrm{~m}\right)$ and one having a very small negative sensible heat flux $(H=-0.001$ $\mathrm{K} \mathrm{m} \mathrm{s}^{-1}$ ), which is in the acceptable range defined in Basu et al. (2008).

Stationary inflow conditions are taken at 2.5 rotor diameters (D) upstream of the turbines simulated in the main simulations (Sect. 2.2 and averaged over a line of size $2 \mathrm{D}$ in crosswise direction and a period of 60 minutes. These inflow conditions are assumed to be undisturbed, hence far enough from the turbine that induction does not play a role. The most relevant inflow parameters are displayed in Fig. 1, showing comparable wind speed for all simulations and dissimilar atmospheric conditions related to the simulated stratification. A more stable boundary layer, indicated by a larger Obukhov stability parameter $\left(z L^{-1}\right)$, typically has a higher shear $(\alpha)$ and veer $(\partial \alpha)$ and lower turbulence intensity $(T I)$. The spread of the parameters between the main simulations (see Sect. 2.2) in the same boundary layer, indicated by the standard deviation as whiskers in Fig. 1, is small enough to be neglected.

\subsection{Main simulations}

After generating stationary inflow conditions with a precursor simulation, a simulation with one turbine is performed. Information on turbulence characteristics from the precursor simulation is fed to the main simulation by adding a turbulent signal to a fixed mean inflow (turbulence recycling method) far upstream of the turbine. A radiation boundary condition ensures undisturbed outflow downstream of the simulated turbine. The size of the recycling area is equal to the domain size of the precursor simulation and the domain size of the main simulation is only extended in streamwise direction by placing a turbine at $x=6$ $\mathrm{D}$ downstream of the recycling area. Wake data until $x=10 \mathrm{D}$ are used for analysis, but the domain is extended to $x=13 \mathrm{D}$ to eliminate blockage effects. The simulation time consists of 20 minutes spin-up time followed by 60 minutes used for analysis. The simulated turbine is an Actuator Disc Model with Rotation (ADMR) representing a 5MW NREL turbine, having a hub height of $90 \mathrm{~m}$ and a rotor diameter $D$ of $126 \mathrm{~m}$ (Jonkman et al., 2009), as implemented in Dörenkämper et al. (2015). Turbine 


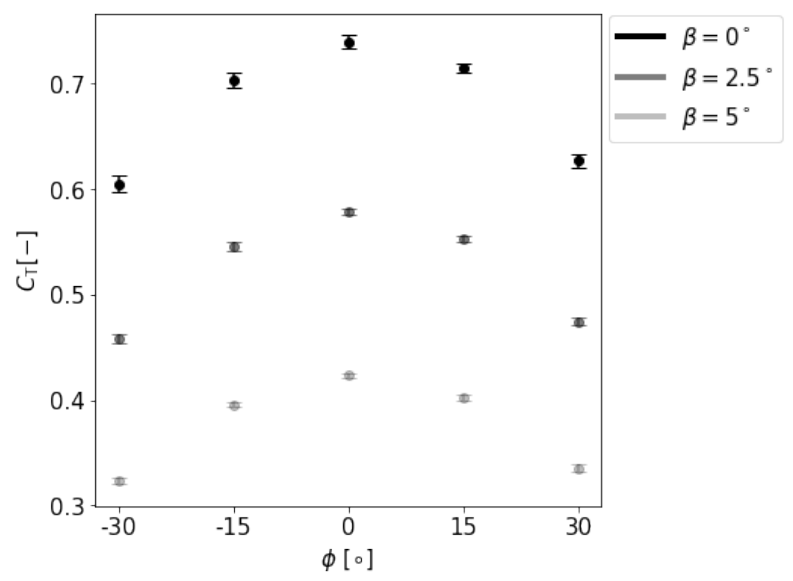

Figure 2. Overview of the effect of yaw angle $\phi$ and pitch angle $\beta$ on thrust coefficient $C_{\mathrm{T}}$. Whiskers indicate the standard deviation between all eight BLs.

yaw angles $(\phi)$ of $-30^{\circ},-15^{\circ}, 0^{\circ}, 15^{\circ}$, and $30^{\circ}$ are simulated, where a positive yaw angle is here defined as a clockwise rotation of the turbine when looking from above. Pitch angles $(\beta)$ of $0^{\circ}, 2.5^{\circ}$, and $5^{\circ}$ are simulated to study the effect of the thrust force on downstream wake characteristics. This adds up to a total of 120 main simulations with one turbine, i.e. eight inflow conditions times five yaw angles times three pitch angles. The effect of $\phi$ and $\beta$ on the thrust coefficient $C_{\mathrm{T}}$ is illustrated in Fig. 2, illustrating that the effect of the turbine yaw angle of the thrust coefficient is symmetric around zero.

The wake is described using the normalized wake deficit, defined as $u_{n d}=\frac{u_{\text {wake }}-u_{\infty}}{u_{\infty, \mathrm{h}}}$, where $u_{\text {wake }}$ represents the observed wind speed in the wake, $u_{\infty}$ the undisturbed inflow $2.5 \mathrm{D}$ upstream at the same height and $u_{\infty, \mathrm{h}}$ the undisturbed inflow at hub height. It is assumed that the advection velocity is constant in streamwise direction (assumption of frozen turbulence) and that the wake behaves as a passive tracer in the ambient wind (Larsen et al., 2008).

\section{Development of the statistical wake steering model}

This section describes the development of the statistical wake steering model (SWSM). Figure 3 displays a flowchart of the training and execution (including testing) procedure of the model. The model is trained with the LES data representing reference inflow conditions (BLs) generated in Sect. 2. From these data, input parameters and key wake steering parameters are deducted.

110 A coefficient matrix is subsequently generated by performing a multi-task Lasso regression. This matrix can be used in the execution (testing) of the model to estimate the key wake steering parameters with new input parameters (e.g. new inflow conditions), which can subsequently be used to produce gridded wake data. 
(a)

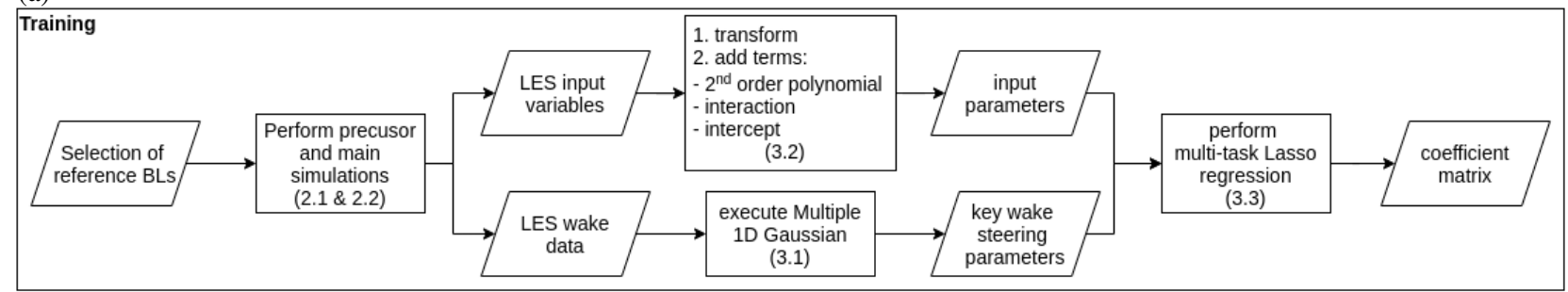

(b)

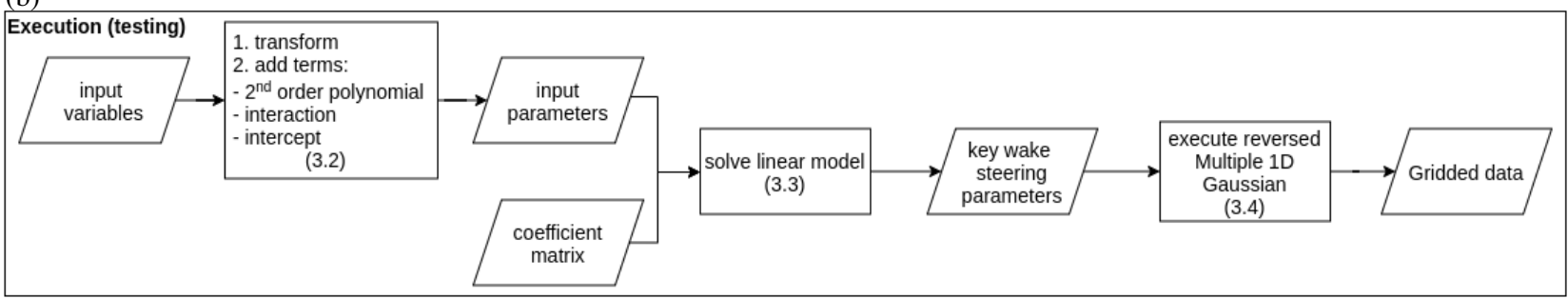

Figure 3. Flowchart describing the training (a) and execution (b) procedure of the statistical wake steering model. Between parenthesis is indicated in what section the process is described. The coefficient matrix generated in (a) is used in (b).

\subsection{Defining key wake steering parameters}

A statistical model will not be able to produce a full multidimensional wake, but rather estimate parameters describing the wake at downstream positions, for instance at one rotor diameter intervals as done here. Since curled wakes are considered, key wake steering parameters are in this study retrieved with the Multiple 1D Gaussian method (Sengers et al., 2020). In the example below, the wake of a turbine with $\mathrm{a}+30^{\circ}$ yaw angle in BL1 at $x=5 \mathrm{D}$ is considered (Fig. $4 \mathrm{a}$ ). This method fits a simple 1D Gaussian at every vertical level $(k=1 \ldots K)$ where information is available to obtain a set of local normalized wake center deficits $\left(\boldsymbol{A}=A_{1} \ldots A_{K}\right)$, wake center positions $\left(\boldsymbol{\mu}=\mu_{1} \ldots \mu_{K}\right)$ and wake widths $\left(\boldsymbol{\sigma}=\sigma_{1} \ldots \sigma_{K}\right)$. Subsequently, another Gaussian can be fitted through the local wake center deficits in the vertical (Fig. 4b) to find the overall normalized wake center deficit $\left(A_{z}\right)$ and vertical position with respect to hub height $\left(\mu_{z}\right)$, as well as the vertical extension of the wake $\left(\sigma_{z}\right)$. The local wake center position and width at vertical level $k$ that corresponds to $\mu_{z}$ are subsequently considered as lateral wake center position $\left(\mu_{y}\right)$ relative to the turbine location and wake width $\left(\sigma_{y}\right)$. Next, by fitting a second order polynomial through the local wake center positions between upper and lower tip height (Fig. 4c), one obtains a measure for the curl (coefficient of quadratic term) and tilt (coefficient of linear term) of the wake. An expression for the wake width as function of height is found by repeating this step for the local wake widths (Fig. $4 \mathrm{~d}$ ) to obtain coefficients $s_{a}$ and $s_{b}$. After this procedure, the wake can be described by the set of dimensionless parameters displayed in Table 2.

Note that this method cannot accurately capture the splitting of the wake in two separate cells, which might occur under strong veer as discussed in Vollmer et al. (2016). Such cases will result in inaccurate values for the key wake steering parameters and should be filtered out before applying the statistical model described in Sect. 3.3. 

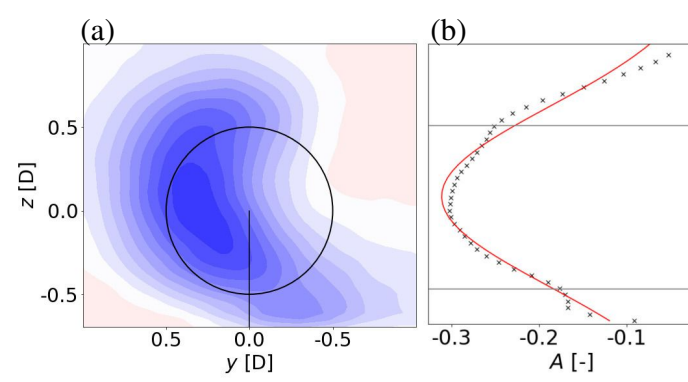

(c)
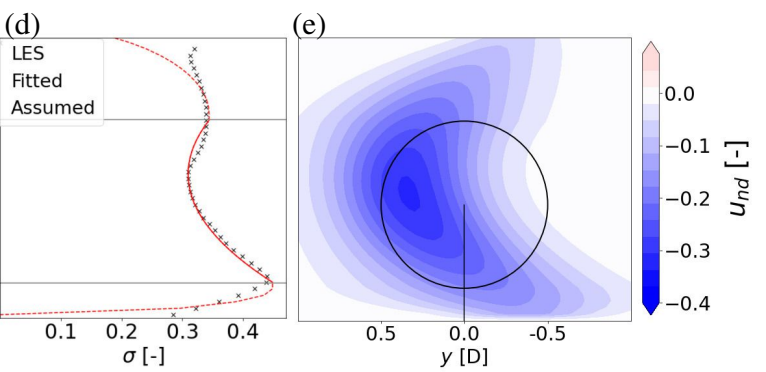

Figure 4. Exemplary figures (BL1, $\phi=+30^{\circ}, x=5 \mathrm{D}$ ) illustrating the key wake steering parameters. (a) Normalized wake deficit cross section (contour) of original LES data. (b) The local normalized wake center deficits $\boldsymbol{A}$, (c) local wake center positions $\boldsymbol{\mu}$, (d) local wake widths $\sigma$. Black crosses indicate LES, red solid lines the relation fitted in according to the Multiple 1D Gaussian method (Sect. 3.1) and red dashed lines the assumed continuation in the reversed Multiple 1D Gaussian composition method (Sect. 3.4). e) Cross section (contour) of the normalized wake deficit after applying the reversed Multiple 1D Gaussian composition method.

Table 2. Defined dimensionless key wake steering parameters. The normalized wake deficit is computed as described in Sect. 2.2. All length parameters are nondimensionalized by the rotor diameter $\mathrm{D}$.

\begin{tabular}{ll}
\hline Scalar Parameter & Symbol \\
\hline Amplitude normalized wake deficit & $A_{z}$ \\
Lateral wake center displacement & $\mu_{y}$ \\
Vertical wake center displacement & $\mu_{z}$ \\
Width wake center height & $\sigma_{y}$ \\
Vertical extend & $\sigma_{z}$ \\
Curl & $c u r l$ \\
Tilt & $t i l t$ \\
Quadratic wake width parameter & $s_{a}$ \\
Linear wake width parameter & $s_{b}$
\end{tabular}

\subsection{Input parameters}

A regression model (Sect. 3.3) is used to estimate the key wake steering parameters in Table 2. A set of measurable inflow and turbine variables is used as input parameters, which are made dimensionless to make the model more universally applicable, at least within the variability found between the simulations in this study. This set of parameters is presented in Table 3 .

Although these input parameters might all have their own isolated effect on the wake propagation, they are heavily correlated in LES as shown in Fig. 5. One can identify several highly correlated clusters, representing 1) yaw [ $\phi]$, 2) atmospheric inflow $\left[\delta \alpha, \alpha, z L^{-1}, T I\right]$ and 3$)$ turbine variables $\left[C_{\mathrm{T}}, C_{\mathrm{Q}}, T S R\right]$. Note that wind speed is not included, since it is approximately constant in all simulations and correlated with both inflow and turbine parameters. Because of the highly correlated clusters, it is hypothesized that one is able to achieve reasonable accuracy in estimating key wake steering parameters with the regression 
https://doi.org/10.5194/wes-2021-43

Preprint. Discussion started: 21 May 2021

(c) Author(s) 2021. CC BY 4.0 License.

Table 3. Set of dimensionless input parameters. dir is the wind direction $\left[{ }^{\circ}\right], z$ is the height above the surface $[\mathrm{m}] \overline{u_{\mathrm{h}}}$ and $\sigma_{u_{\mathrm{h}}}$ are the mean and standard deviation of the wind speed at hub height $\left[\mathrm{m} \mathrm{s}^{-1}\right], u_{e f f}$ is rotor effective wind speed $\left[\mathrm{m} \mathrm{s}^{-1}\right], T$ is thrust [N], $Q$ is torque [N

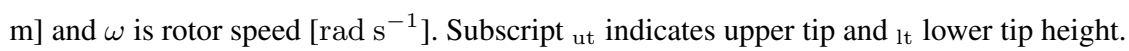

\begin{tabular}{lll}
\hline Variable & Symbol & Calculated \\
\hline Turbine yaw angle & $\phi$ & $\phi$ \\
Veer & $\delta \alpha$ & $d i r_{\mathrm{ut}}-d i r_{\mathrm{lt}}$ \\
Shear & $\alpha$ & $\ln \frac{u_{\mathrm{ut}}}{u_{\mathrm{tt}}} / \ln \frac{z_{\mathrm{ut}}}{z_{\mathrm{lt}}}$ \\
Obukhov stability parameter & $z L^{-1}$ & $2.5 / L$ \\
Turbulence intensity & $T I$ & $\sigma_{u_{\mathrm{h}}} / \overline{u_{\mathrm{h}}}$ \\
Thrust coefficient & $C_{\mathrm{T}}$ & $T /\left(0.5 \rho u_{\text {eff }}^{2} \pi(D / 2)^{2}\right)$ \\
Torque coefficient & $C_{\mathrm{Q}}$ & $Q /\left(0.5 \rho \pi u_{\text {eff }}^{2}(D / 2)^{3}\right)$ \\
Tip speed ratio & $T S R$ & $\omega(D / 2) / u_{\mathrm{h}}$
\end{tabular}

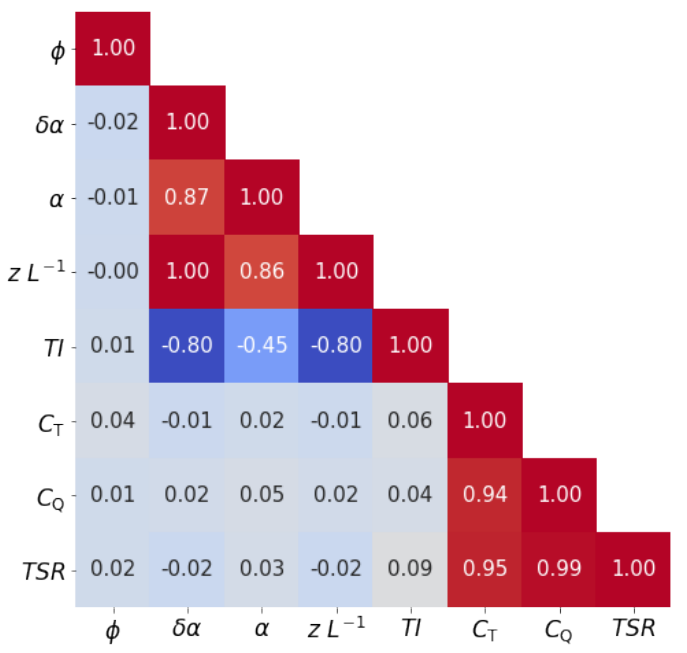

Figure 5. Correlation matrix of the dimensionless input parameters in LES. Colors indicate a positive (red) or negative (blue) correlation

model as long as each cluster is represented. This would give the user freedom to choose parameters based on preference and availability.

The regression model introduced in Sect. 3.3 is linear. To include nonlinear relations, the input variables can be transformed using reciprocal $\left(f(x)=x^{-1}\right)$, exponential $\left(f(x)=e^{x}\right)$, logarithmic $(f(x)=\ln (x))$ or square root $\left.(f(x)=\sqrt{x})\right)$ transformations. All these transformations have been tested in the optimization described in Sect. 3.5. In addition to the transformed 
variables, second-order polynomial and interaction terms are added, as well as an intercept (unity), extending the original set of three input variables to ten input parameters.

\subsection{Regression model}

Since the LES data set has a relatively small sample size, a linear model is chosen as they perform well on small sample sizes, reduce the risk of overfitting compared to more complex Machine Learning models and are highly interpretable (Hastie et al., 2009).

The regression is formulated as a linear model in matrix form

$\underset{(n \times d)}{\boldsymbol{Y}}=\underset{(n \times p)}{\boldsymbol{X}} \times \underset{(p \times d)}{\boldsymbol{B}}$.

which estimates the output variable $\boldsymbol{Y}$ based on the design matrix $\boldsymbol{Y}$ and coefficient matrix $\boldsymbol{B}$. Matrix dimensions indicated in Eq. (1) represent the sample size $n$, number of downstream distances $d$ and number of input parameters $p$. Note that $p$ contains the transformed variables, their second-order and interaction terms, as well as intercepts. Since these parameters are highly correlated and not all relevant, the coefficients are determined based on a Lasso regression method as introduced by Tibshirani (1996). This guarantees a shrinkage of the number of variables through an regularization parameter found by cross-validation. Additionally, it is desired that the same set of input parameters is used to estimate the output variable at all downstream distances. This is guaranteed in the multi-task Lasso method introduced by Obozinski et al. (2006), which is implemented in the multi-task Lasso algorithm from the scikit-learn Python library (Pedregosa et al., 2011). See Appendix A for further explanation.

Whereas the training is more complex than Ordinary Least Squares fitting, the predictions in the testing are generated through simple matrix multiplication as shown in Eq. (1). The algorithm is therefore highly interpretable, easy to implement and computational inexpensive.

\subsection{Wake composition: reversed Multiple 1D Gaussian}

The coefficient matrix $\boldsymbol{B}$ can be used to estimate the key wake steering parameters in Table 2 from new input parameters. This information can be used to compose a vertical cross section of the wake deficit using a methodology that is very similar to the reverse of the Multiple 1D Gaussian method described in Sect. 3.1. IN this section, the key wake steering parameters from the example in Figure 4 are used to illustrate this composition method, hence no predictions with the coefficient matrix $\boldsymbol{B}$ are done. For a fair comparison of the composed wake to the original LES, a resolution identical to the LES ( $\Delta=5 \mathrm{~m})$ is chosen. First, using the information from the vertically fitted Gaussian $\left(A_{z}, \mu_{z}, \sigma_{z}\right)$, one can compute the amplitude of the normalized wake deficit $\hat{\boldsymbol{A}}$ at each height $(\mathrm{k}=1 . . \mathrm{K})$ by simply filling out the Gaussian function (Fig. $4 \mathrm{~b})$. This information is then considered the local wake center deficit at every height.

Next, to identify local wake center positions $\hat{\mu}$, one can calculate the distance to the origin (turbine location) by filling out the simple second order polynomial using the curl and tilt parameters and the wake center location $\left(\mu_{y}, \mu_{z}\right)$. It is assumed that curl continues outside of the rotor area (red dashed line in Fig. 4c). Since this was not included in the fitting of the parameters, 
a deviation is expected.

Similarly, the fitted parameters $s_{a}, s_{b}$ can be used in a second order polynomial to find the set of local wake widths $(\hat{\boldsymbol{\sigma}})$, normalized by the wake width at the height of the wake center $\left(\sigma_{z}\right)$, within the vertical extent of the rotor area. Outside of the rotor area (red dashed line in Fig. 4d), the definition of an ellipse $\left(\frac{\sigma(z)^{2}}{\sigma_{\text {tip }}^{2}}+\frac{\left(z-z_{\text {tip }}\right)^{2}}{\left(z_{\text {tip }}-z_{\mathrm{base}}\right)^{2}}=1\right)$ is used to determine the wake width. Here, $\sigma(z)$ indicates the wake width at height $z, \sigma_{\text {tip }}$ the wake width at lower or upper tip height, $z-z_{\text {tip }}$ the distance to tip height and $z_{\text {tip }}-z_{\text {base }}$ the distance from tip height to the surface or wake top. Using information on wake center height $\mu_{z}$ and wake width $\sigma_{y}$, one obtains a set of local wake widths.

Finally, a simple 1D Gaussian can be filled out at every vertical level using the information from $\hat{A}, \hat{\mu}, \hat{\sigma}$, resulting in a two-dimensional grid filled with $u_{n d}$ values. This data can then be plotted in a cross section shown in Fig. 4e. Comparing this composed wake to the original LES in Fig. 4a, one can see that this simplification still contains much of the original information. The shape of the wake is conserved, as well as the displacement of the wake center. The maximum deficit of the composed wake center appears to be slightly larger than in LES. Additionally, in the composition the maximum wake deficit is always in the center (definition of a Gaussian), which is not necessarily true in LES or reality.

\subsubsection{Wake composition validation}

The procedure described in Sect. 3.4 is repeated for all 120 simulations and $1 \mathrm{D} \leq x \leq 10 \mathrm{D}$ at every $\mathrm{D}$. The metric used here to evaluate the accuracy of this method is the percentage error of available power in the rotor area of the composed wake relative to when computed with the original LES wind field $\left(P E[\%]=\left(P_{\text {comp }}-P_{\mathrm{LES}}\right) / P_{\mathrm{LES}} * 100\right)$. A few things can be noted by studying the results shown in Fig. 6 . The composition shows a large systematic positive bias in the near wake $(x \leq 3$ D). This is due to the so-called double bell shape of the near wake, with a speed up region around hub height. When attempting to fit this with a simple 1D Gaussian, the deficit in the rotor area is underestimated, resulting in a positive percentage error. For this reason, the near wake will be excluded from analysis in the remainder of this work. Further downstream $(x \geq 8 \mathrm{D})$ a small negative systematic bias can be identified, which is due to the 'top head' shape of the wake deficit as a result of temporal averaging. This is not captured by a Gaussian function and will on average result in an overestimation of the wake deficit amplitude. The large (negative) outliers typically indicate cases where the wake does not have a Gaussian shape, such as the separation in two cells under strong veer. The median error in the region $4 \mathrm{D} \leq x \leq 10 \mathrm{D}$ is, however, smaller than $1 \%$.

\subsection{Optimization}

Numerous combinations of input parameters, consisting of different variables and their transformations, are possible. In order to find the most accurate solution, an optimization procedure needs to be executed. Here, the goal is to minimize the absolute percentage error of available power over all training data, i.e. all considered simulations and downstream distances ( $4 \mathrm{D} \leq x \leq$ $10 \mathrm{D})$. All combinations of input parameters, one variable per cluster, and transformations have been tested in order to find the optimal solution, denoted oSWSM. Additionally, a second version, denoted cSWSM, is considered that is based on the cost efficient (relatively cheap to obtain) input parameters $\left\{\phi, z L^{-1}, T S R\right\}$. Since the input variables are know, the optimization 


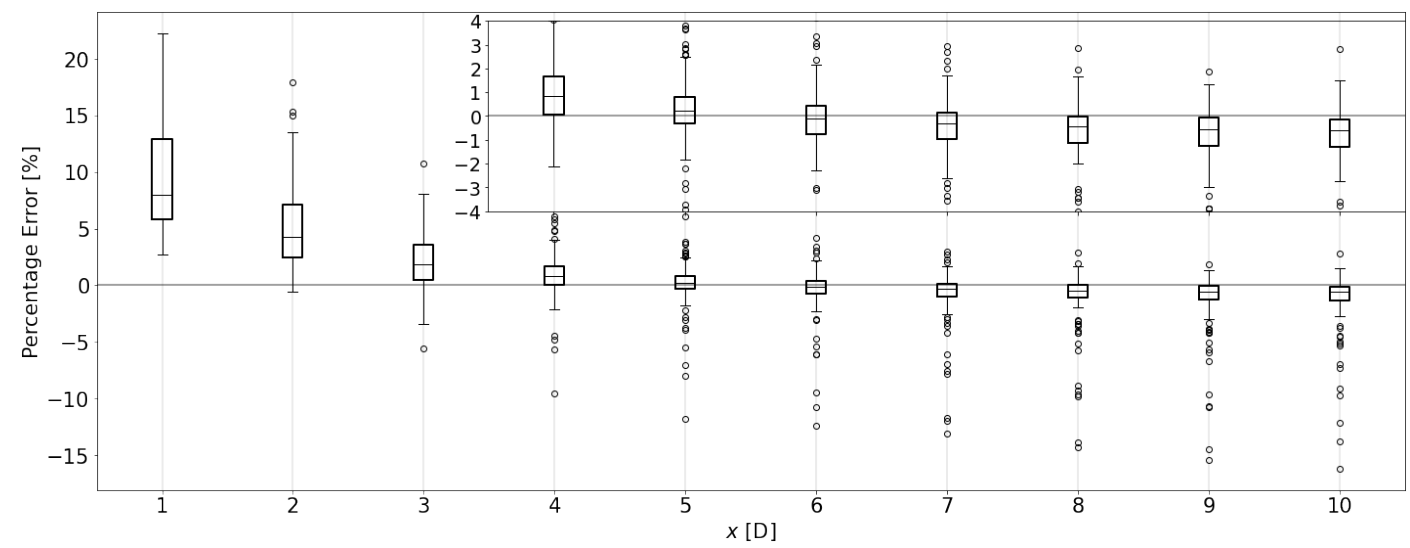

Figure 6. Accuracy of the wake composition procedure expressed as a percentage error of available power of a virtual downstream turbine. At each downstream distance, data from all 120 simulations are considered.

procedure is limited to finding the optimal transformations for these variables. Both oSWSM and cSWSM will be considered in Sect. 4.

\subsection{Benchmark models}

SWSM is benchmarked against the Gaussian (GAUS) and the Gaussian-Curl Hybrid (GCH) models present in version 2.2.2 of the FLORIS framework (NREL, 2020). Although secondary steering is not studied here, the GCH is still included because of its incorporation of initial wake deflection and the added wake recovery term. Both models share the same tuning parameters for the far wake onset $\left(\alpha_{\text {floris }}, \beta_{\text {floris }}\right)$ and wake recovery rate $\left(k_{a, \text { floris }}, k_{b \text {,floris }}\right)$, in which subscript floris is added to avoid confusion with other parameters. Analogous to the training of SWSM discussed in Sect. 3.5, the values of the tuning parameters is determined by minimizing the APE of available power over all considered simulations and downstream distances ( $4 \mathrm{D} \leq x \leq$ 10 D). Information on inflow (e.g. $u_{\mathrm{h}}, T I$ ) is taken from the LES data. The models are trained independently of each other and will therefore have different values for the tuning parameters.

The data used for the tuning include simulations with yaw and pitch angles. FLORIS adjusts the thrust coefficient numerically for yaw angles, but not for pitch angles. For this reason, the thrust coefficient lookup table was adjusted by the ratio $C_{\mathrm{T}, \text { pitch }} / C_{\mathrm{T}, \text { nopitch }}$ found in LES (Fig. 2).

\section{Results}

\subsection{Performance on training data}

This section displays the performance of the statistical wake steering model (SWSM) and the benchmark models when using all 120 simulations as training data. This is done to illustrate the effect of choosing a different set of input parameters on the 
accuracy of SWSM, as well as to highlight the differences between GAUS and GCH. In Sect. 4.2 and 4.3 a validation of the model with testing data will be shown.

Following the optimization procedure as described in Sect. 3.5, the optimal combinations of input parameters, denoted oSWSM, was found to be the set $\left\{\phi, \delta \alpha^{-1}, \ln \left(C_{\mathrm{T}}\right)\right\}$ and the optimal transformations of the cost efficient input parameters, denoted cSWSM, is $\left\{\phi, \sqrt{z L^{-1}}, T S R\right\}$. Figure 7 illustrates the performance of these versions as a function of downstream distance. The accuracy of oSWSM and cSWSM is very comparable with the latter showing a slightly larger spread. However, both show a median percentage error smaller than $2 \%$ and the interquartile range stretches over less than $5 \%$. This suggests that SWSM allows for using different sets of input parameters against minimal loss of accuracy.

To test whether a higher accuracy is achieved when more variables are included, allSWSM uses all (non-transformed) available variables of Table 3 as input. No optimization has been carried out for this test. The high correlation between variables is not an issue due to the use of the regression method described in Sect. 3.3. This results in an accuracy gain closer to the turbine, since the near wake is more dynamic and therefore needs more parameters to explain its variability. Further downstream this effect diminishes, but is still visible. This suggests that adding more variables can indeed lead to a higher accuracy. Figure 8 shows the accuracy of GAUS and GCH when using all simulations for the tuning described in Sect. 3.6. GCH consistently gives a higher power estimate than GAUS due to the added wake recovery term. The median shows a negative bias closer to the turbine for both models, which is opposite to SWSM in Fig. 6. The biases of the benchmark models and oSWSM are of comparable magnitude. Most striking is the variability of the benchmark models that is an order of magnitude larger than that of oSWSM. The reason for this will be systematically evaluated in Sect. 4.2 and 4.3.

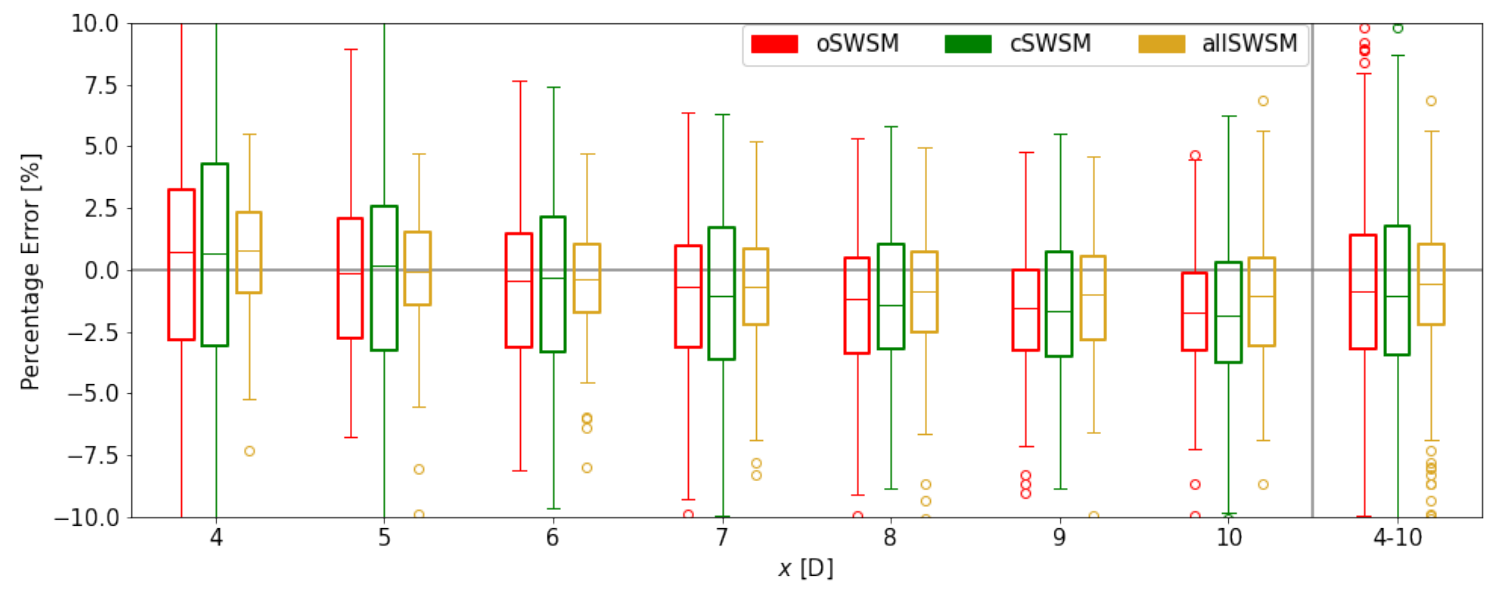

Figure 7. Performance of the statistical model on training data displayed as percentage error of available power. In red oSWSM $\left\{\phi, \delta \alpha^{-1}, \ln \left(C_{\mathrm{T}}\right)\right\}$, in green cSWSM $\left\{\phi, \sqrt{z L^{-1}}, T S R\right\}$ and in blue allSWSM $\left\{\phi, \delta \alpha, \alpha, z L^{-1}, T I, C_{\mathrm{T}}, C_{\mathrm{Q}}, T S R\right\}$. The boxes on the far right (labeled 4-10) include all simulations and all distances. 


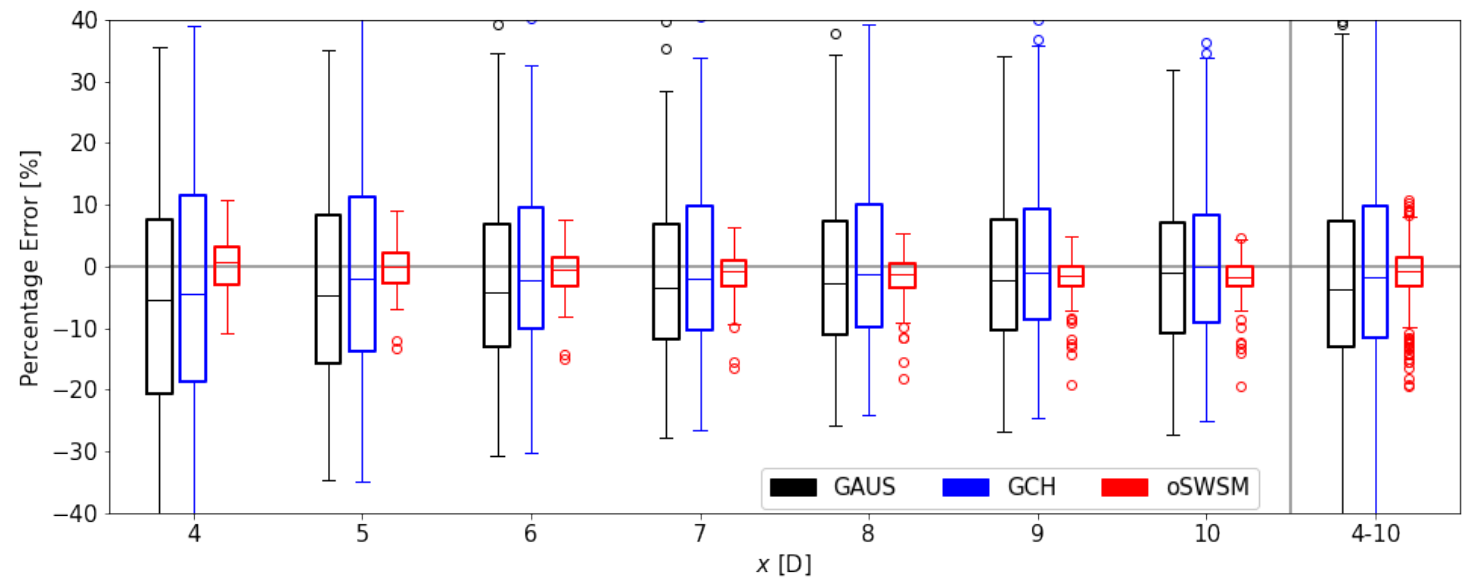

Figure 8. Same as Fig. 7, but for GAUS (black) and GCH (blue). oSWSM (red) is included as a reference.

\subsection{Performance on testing data}

A simple leave-one-out cross-validation technique is used to discuss the performance of SWSM compared to the benchmark models. The models are trained or tuned with seven out of the eight BLs (Fig. 1) and tested on the remaining one representing a new inflow condition. Eight evaluations can therefore be performed, i.e. each BL being tested once. Note that for each evaluation a set of optimal parameters and transformations are determined, which can differ from oSWSM and cSWSM in Fig. 7. Similarly, GAUS and GCH are tuned again, resulting in new values for their tuning parameters. Since the models show similar behavior in relation to the downstream distance as discussed before, here only the collective result over $4 \mathrm{D} \leq x \leq 10 \mathrm{D}$ is discussed.

Figure 9 presents the results of this validation procedure. The shaded areas indicate a significant improvement (green), insignificant difference (yellow) or significant decline (red) of the SWSM accuracy compared to the best performing benchmark model. Statistical significance is determined using an independent Welch's t-test on the absolute percentage error with a p-value $<0.05$. This test assumes a normal distribution, but can deal with unequal variances between data sets.

For most BLs, SWSM shows a significant improvement over GAUS and GCH. The systematic biases (indicated by the medians) are similar for all models in the order of a few percent, but the variability is greatly reduced in SWSM. The main reason for this is that the benchmark models do not include a pitch angle parameter $\beta$. Although the $C_{\mathrm{T}}$ tables in the models are corrected in this study, the tunable parameters do not account for this. To clarify, LES finds a decreasing wake size (in both horizontal and vertical extent) with increasing $\beta$. This is accurately captured by SWSM, but GAUS and GCH produce a wake of similar size independent of $\beta$ or $C_{\mathrm{T}}$. The inclusion of this effect is a notable improvement of SWSM. Arguably, this is currently not a major disadvantage of the benchmark models as turbines tend to operate without derating $\left(\beta=0^{\circ}\right)$. However, this might get more important in the future with control strategies such as axial induction control (e.g. Corten and Schaak, 2003; van der 


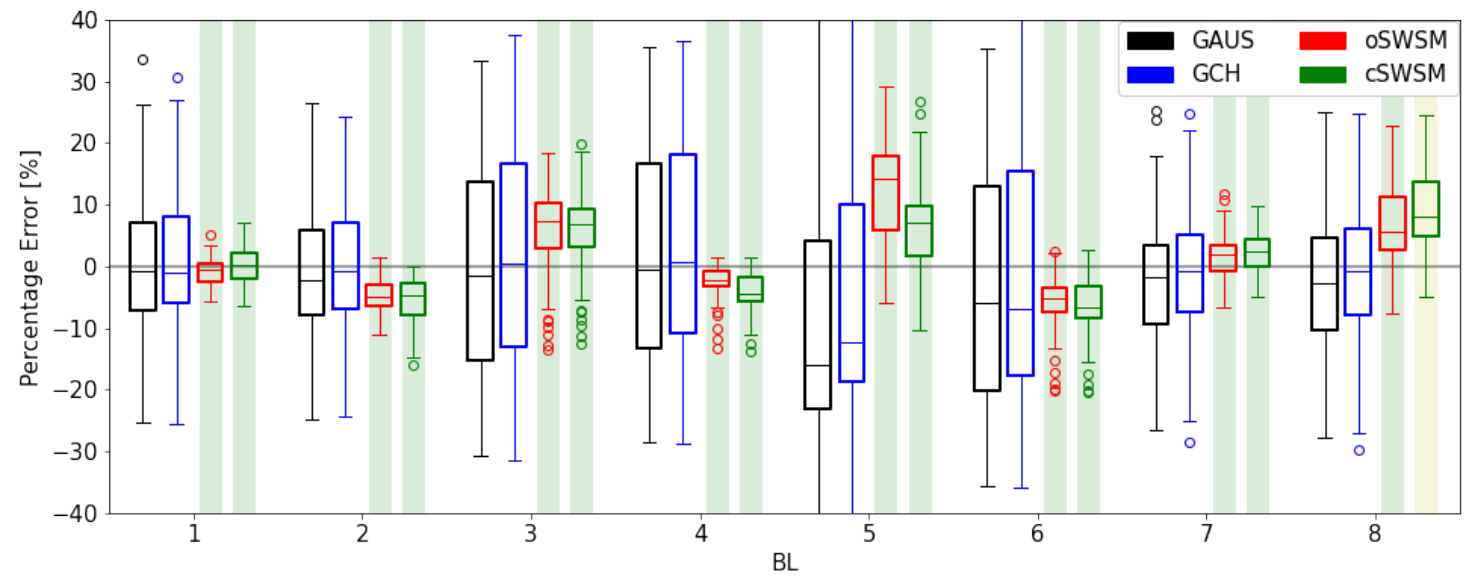

Figure 9. Performance of GAUS (black), GCH (blue), oSWSM (red) and cSWSM (green) using a leave-one-out cross-validation technique. Performance is displayed as a percentage error of available power. Each box includes data from 15 main simulations and $4 \mathrm{D} \leq x \leq 10 \mathrm{D}$. The shaded areas indicate a significant improvement (green), insignificant difference (yellow) or significant decline (red) of the accuracy of SWSM compared to the benchmark models.

Hoek et al., 2019).

Furthermore, BL5 contains the worst results for all models. Figure 1 indicates that this is an extreme case as it has the highest Obukhov stability parameter and veer along with the lowest turbulence intensity. This is problematic for the models, since it is an inflow condition unlike anything it was trained for.

Figure 9 also shows that oSWSM and cSWSM generally have a very comparable performance, where the former generally has a slightly higher accuracy. However, the differences are small, illustrating the flexibility that SWSM provides by allowing the user to choose the input parameters.

\subsection{Operation without derating}

For a fair comparison between SWSM and the benchmark models, this section only considers simulations representing operation without derating the turbine $\left(\beta=0^{\circ}\right)$. The training (selection of parameters for oSWSM and cSWSM) and tuning (tuning parameters of GASU and GCH) has been repeated and the results of the leave-one-out cross-validation technique are displayed in Fig. 10. The variability of the benchmark models in (near) neutral conditions (BL 1, 2, 7 and 8) decreases considerably, but generally SWSM still produces significantly more accurate results. In (weakly) stable boundary layers (BLs 3 to 6) GAUS and GCH still show a large variability and occasionally a large systematic bias, which is not true for SWSM. These results suggest that SWSM outperforms the benchmark models especially under stable stratifications, those conditions where wake steering is deemed most effective. 
https://doi.org/10.5194/wes-2021-43

Preprint. Discussion started: 21 May 2021

(c) Author(s) 2021. CC BY 4.0 License.

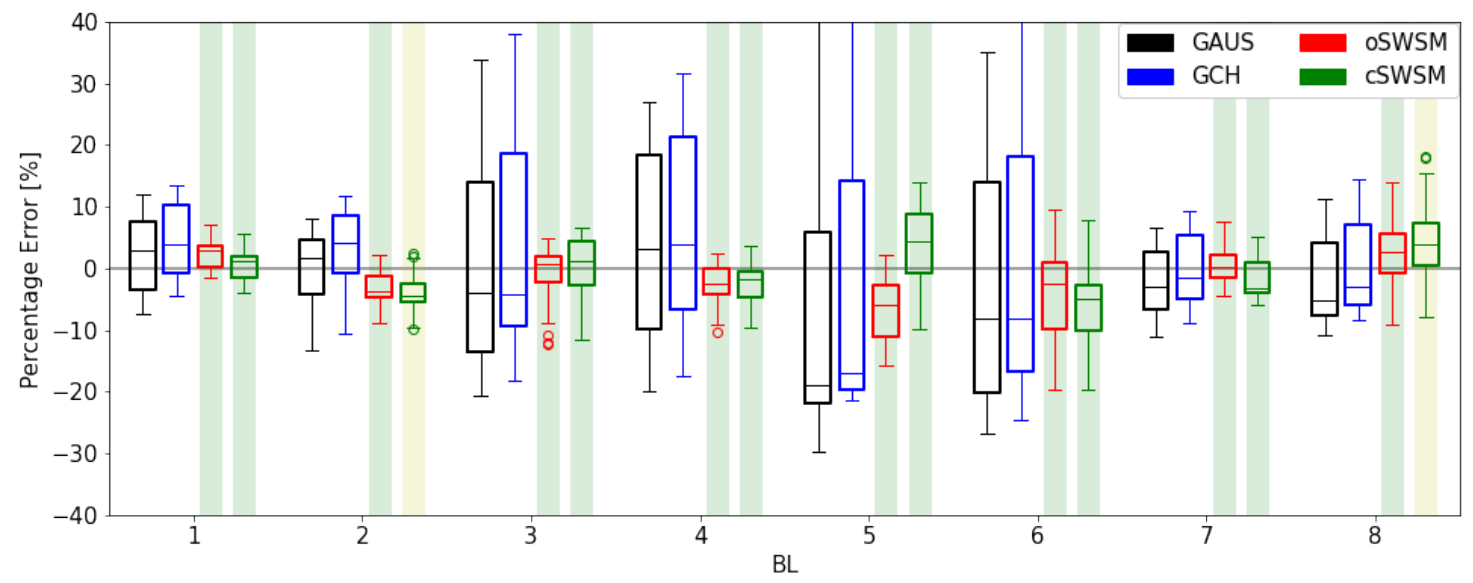

Figure 10. Same as Fig. 9, but only for cases with $\beta=0^{\circ}$, i.e. without derating the turbine.

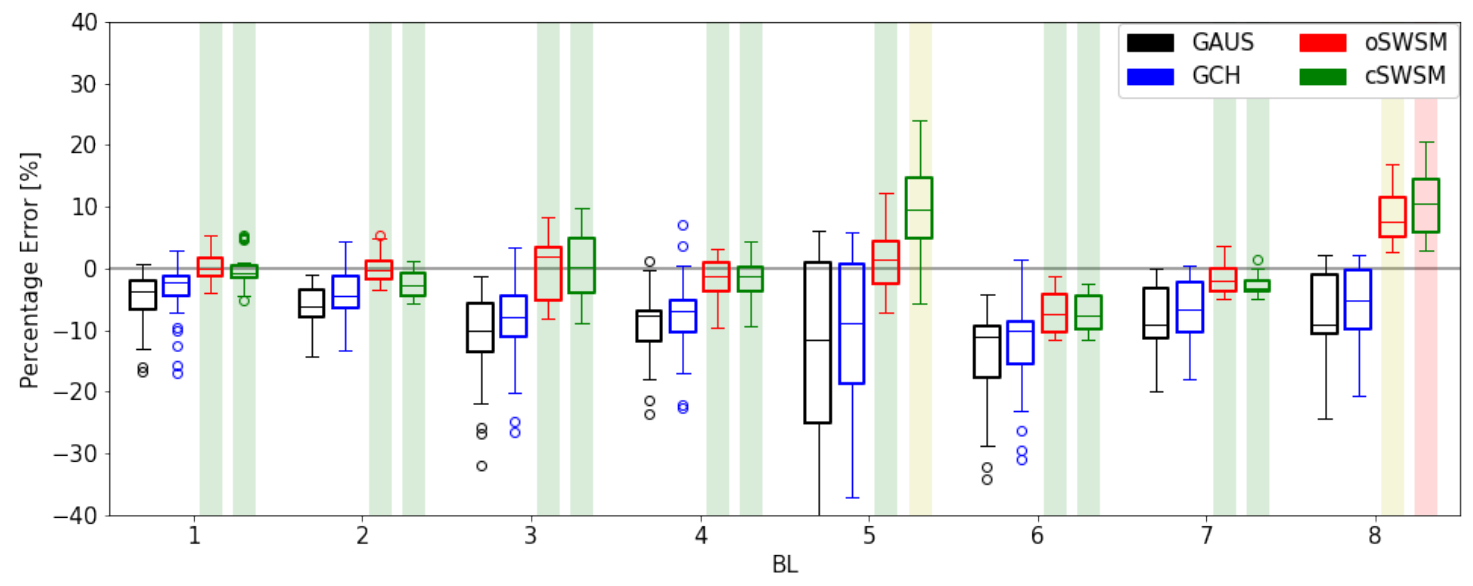

Figure 11. Same as Fig. 10, but for partial wake operation, i.e. with a virtual downstream turbine moved $0.5 \mathrm{D}$ to the left.

Furthermore, the model performance is assessed for partial wake operation. Fig. 11 compares the models when the downstream turbine is moved $0.5 \mathrm{D}$ to the left (from the upstream observer's point of view). Generally, the variability is greatly reduced since the deficit is smaller. The benchmark models display a systematic negative bias in all BLs, which is not true for SWSM. Only BL8 shows a poorer performance of SWSM, but no satisfying explanation has been found why exactly this BL has this behavior.

A case study is displayed in Fig. 12a that presents the LES wind field in a weakly stable boundary layer (BL3). The wake has a clearly defined curl and a wake center left of the hub. The oSWSM wind field in Figure 12b shows that the wake shape and 
(a)

(b)

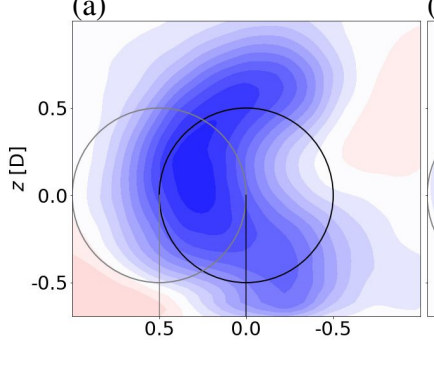

(c)

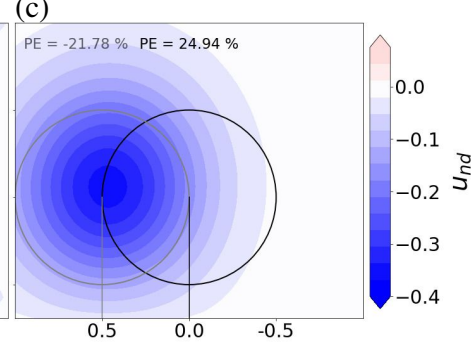

(d)

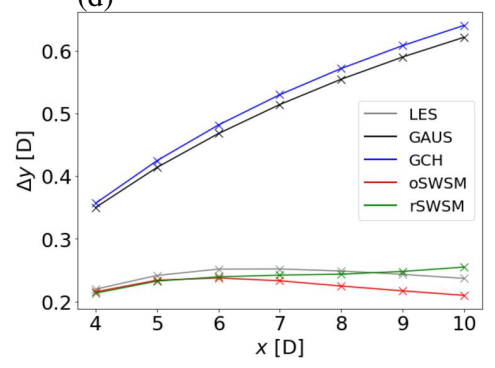

Figure 12. Case study of a turbine in a weakly stable boundary layer (BL3, $\left.\phi=+30^{\circ}, \beta=0^{\circ}\right)$. Cross section of normalized wake deficit (contours) of the LES (a), oSWSM (b) and GAUS (c) at $x=6$ D downstream. (d) Wake center trajectory at $4 \mathrm{D} \leq x \leq 10 \mathrm{D}$.

center position are well presented. The GAUS model (Fig. 12c), however, produces a circular wake shape and a larger wake deflection to the left. The percentage errors indicated in the top of the figure show that SWSM has a high accuracy for both virtual turbines, but GAUS has large biases due to the misplacement of the wake center. Under stable conditions the wind veer is relatively high, adding a crosswise force pointing towards to right above hub height. This force effectively opposes the lateral thrust force component introduced by yaw misalignment pointing to the left, reducing the deflection of the wake. The opposite is true for negative yaw angles, where wake deflection is enhanced by veer. This asymmetry has been pointed out in Fleming et al. (2015); Vollmer et al. (2016); Sengers et al. (2020). This effect is implicitly included in SWSM, but not in the benchmark models. Figure 12d illustrates that these models show an ever further deflecting wake, whereas the SWSM settles at a smaller lateral displacement corresponding to LES. This does not only explain the negative bias of the benchmark models in Fig. 11, but also their larger spread observed in Fig. 10. This result strengthens the previous indication that SWSM is superior under stable stratifications.

\section{Discussion}

Although the results in Sect. 4 are encouraging, the current limitations of a statistical model need further exploration. The model is sensitive to the data used for training, and encountering conditions that were not covered can result in large errors, as illustrated by the strongly stable BL5 in Fig. 9. In this study, large eddy simulation data were used to train the model, the generation of which is computationally expensive. As a consequence, the amount of training data was limited and the performance of the model is subject to what conditions were sampled. Here, boundary layers with one reference wind speed were systematically sampled to investigate the performance of the model under different atmospheric conditions. However, one could examine what conditions occur most frequently in the field and sample accordingly. Alternatively, one could mainly target those conditions that are deemed most valuable, i.e. conditions under which high power gains are expected or the risk of losses due to erroneous yawing is high. Especially since the number of cases that can be generated numerically is limited, employing a more refined sampling method than used in this study is encouraged. Furthermore, since the statistical model is sensitive to training data, its direct applicability to other locations and turbine types is questionable. This can be problematic, 
Table 4. Model run time [ms] when simulating seven (4 D $\leq x \leq 10 \mathrm{D})$ and one $(x=6 \mathrm{D})$ downstream distances expressed as mean \pm standard deviation over 40 iterations.

\begin{tabular}{lll}
\hline$x[\mathrm{D}]$ & $\mathbf{4 - 1 0}$ & $\mathbf{6}$ \\
\hline GAUS & $58 \pm 2$ & $19 \pm 1$ \\
GCH & $88 \pm 2$ & $32 \pm 1$ \\
SWSM & $81 \pm 4$ & $13 \pm 3$
\end{tabular}

as generating LES data for each turbine and location is not feasible. However, when properly trained, the accuracy of SWSM is expected to be significantly higher than that of analytical models, as it is specifically trained for a certain situation. Consideration of measured data from the field of wind tunnel might offer potential, although an appropriate measurement strategy and duration need to be explored.

315 If desired, further development of the model is needed to include the near wake, which can for instance be done by including the super-Gaussian description introduced by Blondel and Cathelain (2020). Additionally, an extension from a two-turbine setup to a wind farm could be desired. This could be achieved by for instance applying the superposition principle as done in GAUS and GCH, although the accuracy of SWSM under disturbed inflow needs attention.

Lastly, a simple evaluation of computational costs has been carried out to ensure that SWSM is sufficiently computationally efficient. The speed test comprises of producing cross sections downstream of the turbine and therefore excludes the computational resources needed to generate the LES data and to train or tune the models. This test was executed on a laptop running Ubuntu 20.04.1 with eight 1.80GHz Intel i7-8550U CPU's and 8 GB RAM, having a minimum number of processes running in the background. All files containing relevant information, such as inflow variables, were stored locally at the same location. Run times are given as an average and standard deviation over 40 iterations, representing all simulations with $\beta=0^{\circ}$, such that no adjustment of the benchmark's thrust coefficient lookup table is needed. Table 4 shows that when producing results for the whole region considered in this study ( $\mathrm{D} \leq x \leq 10 \mathrm{D}$ ), the run time of SWSM is comparable to GCH and slightly higher than GAUS. When simulating only one downstream distance, for instance exactly where a turbine is located, SWSM performs similarly to GAUS. These results suggest that SWSM is quick enough to be used for controlling purposes.

\section{Conclusions}

This study explores the potential of a statistical wake steering model that is data-driven, but retains a high degree of physical interpretation. After training with large eddy simulation data, a model consisting of only linear equations is able to accurately describe the curled wake in terms of trajectory, shape and available power. It uses measurable inflow and turbine variables as input parameters and although an optimal set of parameters is found, it allows for choosing different, possibly more cost efficient, input parameters against a minimal loss of accuracy. In a benchmark against the Gaussian and Gaussian-Curl Hybrid models, the statistical wake steering model generally shows a significant improvement in accuracy. In particular it performs better under derated operating conditions and stable atmospheric stratifications, since it implicitly includes the effect of turbine 
https://doi.org/10.5194/wes-2021-43

Preprint. Discussion started: 21 May 2021

(c) Author(s) 2021. CC BY 4.0 License.

(c) (i)

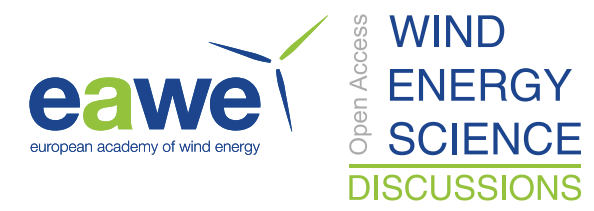

derating on wake size, and the effect of veer on wake shape and center position. Although the results are encouraging, the sensitivity of a statistical model to training data needs further investigation. This includes refining the methodology to sample atmospheric conditions and investigating the model's applicability to other locations and turbines.

340 Code availability. The statistical wake steering model, including a short tutorial, is available for download at: https://github.com/LuukSengers/SWSM 
https://doi.org/10.5194/wes-2021-43

Preprint. Discussion started: 21 May 2021

(c) Author(s) 2021. CC BY 4.0 License.

\section{Appendix A: Multi-task Lasso algorithm}

The original Lasso implementation from Tibshirani (1996) seeks to find the coefficients $B$ based on

$\underset{B}{\operatorname{argmin}} \sum_{n}\left(y_{n}-\sum_{p} x_{n p} B_{p}\right)^{2}+\lambda \sum_{p}\left|B_{p}\right|$.

in which $n$ is the sample size and $p$ the input parameter. It uses the regularization parameter $\lambda$, leading to sparse coefficients for the coefficient vector $B$. The multi-task setting from Obozinski et al. (2006) extends the Lasso regression to estimate $d$ (distance downstream) outputs simultaneously, penalizing the blocks of coefficients over the tasks. The loss function is therefore extended and finds the coefficient matrix $B$ based on

$\underset{B}{\operatorname{argmin}} \sum_{d} \sum_{n}\left(y_{n d}-\sum_{p} x_{n p} B_{p d}\right)^{2}+\lambda \sum_{p} \sqrt{\sum_{d}\left(B_{p d}\right)^{2}}$.

In contrast to Eq. (A1), the multi-task Lasso implementation does not only penalize the single coefficients, but also the blocks of coefficients over all tasks represented by the Euclidean norm. Note that if $d=1$, Eq. (A2) reduces to the standard Lasso estimate of Eq. (A1).

An exemplary result is illustrated in Fig. A1. Whereas the original Lasso model selects a new set of variables for each distance, the multi-task Lasso always takes the same set. This makes physically more sense and leads to fewer variables in total, therefore reducing the risk of overfitting. The model is optimized using the cyclical descent algorithm implemented in Pedregosa et al. (2011).
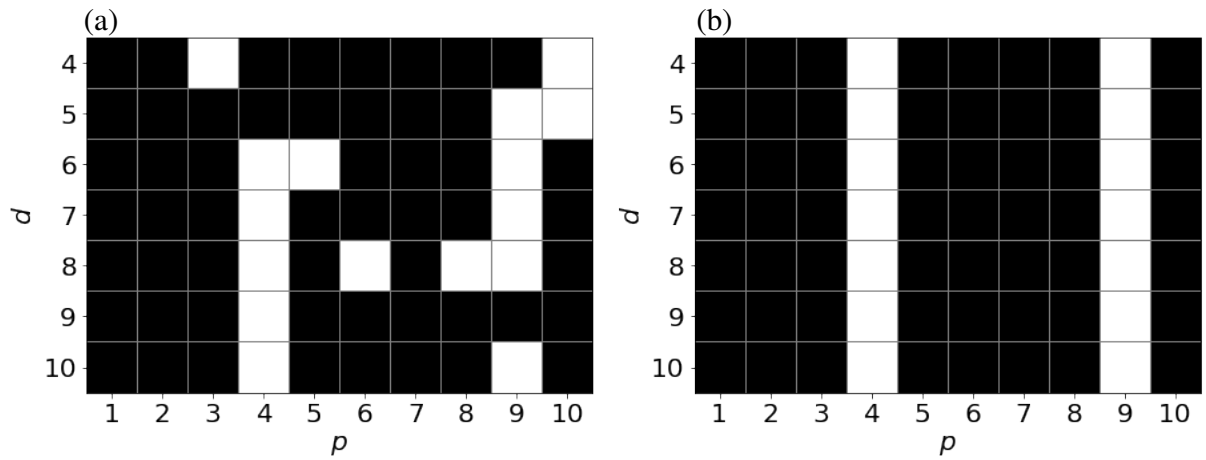

Figure A1. Non-zero elements (black) of $B$ for the output variable $\mu_{y}$ in cSWSM for the original Lasso (a) and multi-task Lasso (b).

Author contributions. BS developed the statistical model, performed the simulations, generated the results and wrote and edited the manuscript. MZ developed the regression model and contributed to writing Sect. 3.3 and Appendix A. PJ tested various tuning strategies for the benchmark models and provided a general understanding of these models. GS provided intensive consultation on the development of the model and the scientific analyses. MK provided general consultation and had a supervisory function. All coauthors reviewed the manuscript. 
https://doi.org/10.5194/wes-2021-43

Preprint. Discussion started: 21 May 2021

(c) Author(s) 2021. CC BY 4.0 License.

(c) (i)

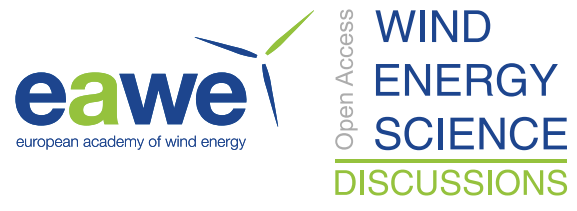

Competing interests. The authors declare that they have no conflict of interest.

Acknowledgements. The authors would like to thank Detlev Heinemann, Paul van der Laan, Lukas Vollmer and Andreas Rott for their contributions in discussions about the direction of this research. David Bastine is thanked for providing insights on feature selection. The presented work has been carried out within the national research project "CompactWind II" (FKZ 0325492H) funded by the Federal Ministry for Economic Affairs and Energy (BMWi) on the basis of a decision by the German Bundestag. The work was partially funded by the Ministry for Science and Culture of Lower Saxony through the funding initiative "Niedersächsisches Vorab". M. Zech has been supported by the Deutsche Bundesstiftung Umwelt (grant no. 20020/667-33/2). Computer resources have been provided by the national research project "Heterogener Hochleistungsrechner für windenergierelevante Meteorologie- und Strömungsberechnungen (WIMS-Cluster)" (FKZ 0324005) funded by the Federal Ministry for Economic Affairs and Energy (BMWi) on the basis of a decision by the German Bundestag. 
https://doi.org/10.5194/wes-2021-43

Preprint. Discussion started: 21 May 2021

(C) Author(s) 2021. CC BY 4.0 License.

\section{References}

Abkar, M. and Porté-Agel, F.: Influence of atmospheric stability on wind-turbine wakes: A large-eddy simulation study, Phys. Fluids, 27, 35 104, https://doi.org/10.1063/1.4913695, 2015.

Annoni, J., Fleming, P., Scholbrock, A., Roadman, J., Dana, S., Adcock, C., Porte-Agel, F., Raach, S., Haizmann, F., and Schlipf, D.: Analysis of control-oriented wake modeling tools using lidar field results, Wind Energ Sci., 3, 819-831, https://doi.org/10.5194/wes-3-819-2018, 2018.

Bartl, J., Mühle, F., Schottler, J., Saetran, L., Peinke, J., Adaramola, M., and Hölling, M.: Wind tunnel experiments on wind turbine wakes in yaw: effects of inflow turbulence and shear, Wind Energ. Sci., 3, 329-343, https://doi.org/10.5194/wes-3-329-2018, 2018.

Bastankhah, M. and Porté-Agel, F.: A new analytical model for wind-turbine wakes, Renew. Energ., 70, 116-123, https://doi.org/10.1016/j.renene.2014.01.002, 2014.

Bastankhah, M. and Porté-Agel, F.: Experimental and theoretical study of wind turbine wakes in yawed conditions, J. Fluid Mech., 806, 506-541, https://doi.org/10.1017/jfm.2016.595, 2016.

Bastankhah, M. and Porté-Agel, F.: Wind farm power optimization via yaw angle control: A wind tunnel study, J. Renew. Sustain. Ener., 11, 023 301, https://doi.org/10.1063/1.5077038, 2019.

Basu, S., Holtslag, A. A. M., Van De Wiel, B. J. H., Moene, A. F., and Steeneveld, G.-J.: An inconvenient "truth" about using sensible heat flux as a surface boundary condition in models under stably stratified regimes, Acta Geophys., 56, 88-99, https://doi.org/10.2478/s11600007-0038-y, 2008.

Beare, R. J. and Macvean, M. K.: Resolution sensitivity and scaling of large-eddy simulations of the stable boundary layer, Bound.-Lay. Meteorol., 112, 257-281, https://doi.org/10.1023/B:BOUN.0000027910.57913.4d, 2004.

Blondel, F. and Cathelain, M.: An alternative form of the super-Gaussian wind turbine wake model, Wind Energ. Sci., 5, 1225-1236, https://doi.org/10.5194/wes-5-1225-2020, 2020.

Bromm, M., Rott, A., Beck, H., Vollmer, L., Steinfeld, G., and Kühn, M.: Field investigation on the influence of yaw misalignment on the propagation of wind turbine wakes, Wind Energy, 21, 1011-1028, https://doi.org/10.1002/we.2210, 2018.

Campagnolo, F., Petrovic, V., Schreiber, J., Nanos, E., Croce, A., and Botasso, C.: Wind tunnel testing of a closed-loop wake deflection controller for wind farm power maximization Recent citations, J. Phys. Conf. Ser., 753, 032 006, https://doi.org/10.1088/17426596/753/3/032006, 2016.

Corten, G. and Schaak, P.: Heat and Flux: Increase of Wind Farm Production by Reduction of the Axial Induction, in: EWEC 2003, 16-19 June, Madrid, Spain, 2003.

Dahlberg, J. and Medici, D.: Potential improvement of wind turbine array efficiency by active wake control (AWC), in: EWEC 2003, 16-19 June, Madrid, Spain, 2003.

Dörenkämper, M., Witha, B., Steinfeld, G., Heinemann, D., and Kühn, M.: The impact of stable atmospheric boundary layers on wind-turbine wakes within offshore wind farms, J. Wind Eng. Ind. Aerod., 144, 146-153, https://doi.org/10.1016/j.jweia.2014.12.011, 2015.

Fleming, P., Gebraad, P. M. O., Lee, S., van Wingerden, J., Johnson, K., Churchfield, M., Michalakes, J., Spalart, P., and Moriarty, P.: Simulation comparison of wake mitigation control strategies for a two-turbine case, Wind Energy, 18, 2135- 2143, https://doi.org/10.1002/we.1810, 2015.

405 Fleming, P., Annoni, J., Shah, J. J., Wang, L., Ananthan, S., Zhang, Z., Hutchings, K., Wang, P., Chen, W., and Chen, L.: Field test of wake steering at an offshore wind farm, Wind Energ. Sci., 2, 229-239, https://doi.org/10.5194/wes-2-229-2017, 2017. 
https://doi.org/10.5194/wes-2021-43

Preprint. Discussion started: 21 May 2021

(C) Author(s) 2021. CC BY 4.0 License.

Fleming, P., Annoni, J., Churchfield, M., Martinez-Tossas, L. A., Gruchalla, K., Lawson, M., and Moriarty, P.: A simulation study demonstrating the importance of large-scale trailing vortices in wake steering, Wind Energ. Sci., 3, 243-255, https://doi.org/10.5194/wes-3-243-2018, 2018.

410 Fleming, P., King, J., Dykes, K., Simley, E., Roadman, J., Scholbrock, A., Murphy, P., Lundquist, J. K., Moriarty, P., Fleming, K., Van Dam, J., Bay, C., Mudafort, R., Lopez, H., Skopek, J., Scott, M., Ryan, B., Guernsey, C., and Brake, D.: Initial Results From a Field Campaign of Wake Steering Applied at a Commercial Wind Farm: Part 1, Wind Energ. Sci., 4, 273-285, https://doi.org/10.5194/wes-4-273-2019, 2019.

Fleming, P., King, J., Dykes, K., Simley, E., Roadman, J., Scholbrock, A., Murphy, P., Lundquist, J. K., Moriarty, P., Fleming, K., van Dam, J., Bay, C., Mudafort, R., Lopez, H., Skopek, J., Scott, M., Ryan, B., Guernsey, C., and Brake, D.: Continued Results from a Field Campaign of Wake Steering Applied at a Commercial Wind Farm: Part 2, Wind Energ. Sci., 4, 945-958, https://doi.org/10.5194/wes-5-945-2020, 2020.

Gebraad, P., Teeuwisse, F., Wingerden, J. v., Fleming, P., Ruben, S., Marden, J., and Pao, L.: Wind plant power optimization through yaw controlusing a parametric model for wake effects—a CFDsimulation study, Wind Energy, 19, 95-114, https://doi.org/10.1002/we.1822, 2016.

Göçmen, T. and Giebel, G.: Data-driven Wake Modelling for Reduced Uncertainties in short-term Possible Power Estimation, J. Phys. Conf. Ser., 1037, 072 002, https://doi.org/10.1088/1742-6596/1037/7/072002, 2018.

Hastie, T., Tibishirani, R., and Friedman, J.: The elements of statistical learning: data mining, inference, and prediction, Springer Science \& Business Media, 2nd edn., 2009.

Howland, M. F., Bossuyt, J., Martínez-Tossas, L. A., Meyers, J., and Meneveau, C.: Wake structure in actuator disk models of wind turbines in yaw under uniform inflow conditions, J. Renew. Sustain. Ener., 8, 043 301, https://doi.org/10.1063/1.4955091, 2016.

Hulsman, P., Juhl Andersen, S., and Göçmen, T.: Optimizing Wind Farm Control through Wake Steering using Surrogate Models based on High Fidelity Simulations, Wind Energ. Sci., 5, 309-329, https://doi.org/10.5194/wes-5-309-2020, 2020.

Jimenez, A., Crespo, A., and Migoya, E.: Application of a LES technique to characterize the wake deflection of a wind turbine in yaw, Wind Energy, 13, 559-572, https://doi.org/10.1002/we.380, 2010.

Jonkman, J., Butterfield, S., Musial, W., and Scott, G.: Definition of a 5-MW Reference Wind Turbine for Offshore System Development, Tech. Rep. NREL/TP-500-38060, National Renewable Energy Laboratory, 2009.

King, J., Fleming, P., King, R., Martínez-Tossas, L., Bay, C., Mudafort, R., and Simley, E.: Controls-Oriented Model for Secondary Effects of Wake Steering, https://doi.org/10.5194/wes-2020-3, in review, 2020.

435 Larsen, G. C., Madsen, H. A., Thomsen, K., and Larsen, T. J.: Wake meandering: A pragmatic approach, Wind Energy, 11, 377-395, https://doi.org/10.1002/we.267, 2008.

Marčiukaitis, M., Žutautaite, I., Martišauskas, L., Jokšas, B., Gecevičius, G., and Sfetsos, A.: Non-linear regression model for wind turbine power curve, Renew. Energ., 113, 732-741, https://doi.org/10.1016/j.renene.2017.06.039, 2017.

Maronga, B., Banzhaf, S., Burmeister, C., Esch, T., Forkel, R., Fröhlich, D., Fuka, V., Gehrke, K. F., Geletič, J., Giersch, S., Gronemeier,

T., Groß, G., Heldens, W., Hellsten, A., Hoffmann, F., Inagaki, A., Kadasch, E., Kanani-Sühring, F., Ketelsen, K., Khan, A., Knigge, C., Knoop, H., Krč, P., Kurppa, M., Maamari, H., Matzarakis, A., Mauder, M., Pallasch, M., Pavlik, D., Pfafferott, J., Resler, J., Rissmann, S., Russo, E., Salim, M., Schrempf, M., Schwenkel, J., Seckmeyer, G., Schubert, S., Sühring, M., Von Tils, R., Vollmer, L., Ward, S., Witha, B., Wurps, H., Zeidler, J., and Raasch, S.: Overview of the PALM model system 6.0, Geosci. Model Dev., 13, 1335-1372, https://doi.org/10.5194/gmd-13-1335-2020, 2020. 
https://doi.org/10.5194/wes-2021-43

Preprint. Discussion started: 21 May 2021

(C) Author(s) 2021. CC BY 4.0 License.

Martínez-Tossas, L. A., Annoni, J., Fleming, P. A., and Churchfield, M. J.: The aerodynamics of the curled wake: a simplified model in view of flow control, Wind Energ. Sci., 4, 127-138, https://doi.org/10.5194/wes-4-127-2019, 2019.

Messner, J. W. and Pinson, P.: Online adaptive lasso estimation in vector autoregressive models for high dimensional wind power forecasting, Int. J. Forecasting, 35, 1485-1498, https://doi.org/10.1016/j.ijforecast.2018.02.001, 2019.

Niayifar, A. and Porté-Agel, F.: Analytical modeling of wind farms: A new approach for power prediction, Energies, 9, 741, https://doi.org/10.3390/en9090741, 2016.

NREL: FLORIS. Version 2.2.2, https://github.com/NREL/floris, 2020.

Obozinski, G., Taskar, B., and Jordan, M.: Multi-task feature selection, Tech. Rep., Statistics Department, UC Berkeley, 2006.

Pedregosa, F., Varoquaux, G., Gramfort, A., Michel, V., Thirion, B., Grisel, O., Blondel, M., Prettenhofer, P., Weiss, R., Dubourg, V., Vanderplas, J., Passos, A., Cournapeau, D., Brucher, M., Perrot, M., and Duchesnay, E.: Scikit-learn: Machine Learning in Python, J. Mach. Learn. Res., 12, 2825-2830, 2011.

Rott, A., Doekemeijer, B., Seifert, J. K., Van Wingerden, J.-W., and Kühn, M.: Robust active wake control in consideration of wind direction variability and uncertainty, Wind Energ. Sci., 3, 869-882, https://doi.org/10.5194/wes-3-869-2018, 2018.

Schottler, J., Hölling, A., Peinke, J., and Hölling, M.: Brief Communication: On the influence of vertical velocity profiles on the combined power output of two model wind turbines in yaw, Wind Energ. Sci., 2, 439-442, https://doi.org/10.5194/wes-2-439-2017, 2017.

Schreiber, J., Bottasso, C. L., Salbert, B., and Campagnolo, F.: Improving wind farm flow models by learning from operational data, Wind Energ. Sci., 5, 647-673, https://doi.org/10.5194/wes-5-647-2020, 2020.

Sengers, B. A. M., Steinfeld, G., Heinemann, D., and Kühn, M.: A new method to characterize the curled wake shape under yaw misalignment, J. Phys. Conf. Ser., 1618, 062 050, https://doi.org/10.1088/1742-6596/1618/6/062050, 2020.

Simley, E., Fleming, P., and King, J.: Design and analysis of a wake steering controller with wind direction variability, Wind Energ. Sci., 5, 451-468, https://doi.org/10.5194/wes-5-451-2020, 2020.

Stathopoulos, C., Kaperoni, A., Galanis, G., and Kallos, G.: Wind power prediction based on numerical and statistical models, J. Wind Eng. Ind. Aerod., 112, 25-38, https://doi.org/10.1016/j.jweia.2012.09.004, 2013.

Ti, Z., Deng, X. W., and Yang, H.: Wake modeling of wind turbines using machine learning, Appl. Energ., 257, 114025, https://doi.org/10.1016/j.apenergy.2019.114025, 2020.

Tibshirani, R.: Regression Shrinkage and Selection Via the Lasso, J. Roy. Stat. Soc. B. Met., 58, 267-288, https://doi.org/10.1111/j.25176161.1996.tb02080.x, 1996.

van der Hoek, D., Kanev, S., Allin, J., Bieniek, D., and Mittelmeier, N.: Effects of axial induction control on wind farm energy production A field test, Renew. Energ., 140, 994-1003, https://doi.org/10.1016/j.renene.2019.03.117, 2019.

Vollmer, L., Steinfeld, G., Heinemann, D., and Kühn, M.: Estimating the wake deflection downstream of a wind turbine in different atmospheric stabilities: an LES study, Wind Energ. Sci., 1, 129-141, https://doi.org/10.5194/wes-1-129-2016, 2016.

Wagenaar, J., Machielse, L., and Schepers, J.: Controlling Wind in ECN's Scaled Wind Farm, in: Proceeding of the EWEA Annual Meeting, 16-19 April, Copenhagen, Denmark, 2012.

Zahle, F. and Sørensen, N. N.: Overset grid flow simulation on a modern wind turbine, in: AIAA 26th Applied Aerodynamic Conference, 18-21 August, Honolulu, Hawaii, USA, https://doi.org/10.2514/6.2008-6727, 2008. 